\title{
A novel in situ simulation framework for introduction of a new technology: the 3-Act-3-Debrief model
}

Lisa T. Barker ${ }^{1,2}$, William F. Bond ${ }^{1,2}$, Andrew L. Vincent ${ }^{2}$, Kimberly L. Cooley ${ }^{1 *} \mathbb{D}$, Jeremy S. McGarvey ${ }^{1}$, John A. Vozenilek ${ }^{1,2}$ and Emilie S. Powell ${ }^{3}$

\begin{abstract}
Background: New technologies for clinical staff are typically introduced via an "in-service" that focuses on knowledge and technical skill. Successful adoption of new healthcare technologies is influenced by multiple other factors as described by the Consolidated Framework in Implementation Research (CFIR). A simulation-based introduction to new technologies provides opportunity to intentionally address specific factors that influence adoption.

Methods: The new technology proposed for adoption was a telehealth cart that provided direct video communication with electronic intensive care unit (elCU) staff for a rural Emergency Department (ED). A novel 3-Act-3Debrief in situ simulation structure was created to target predictive constructs from the CFIR and connect debriefing to specific workflows. The structure and content of the simulation in relation to the framework is described. Participants completed surveys pre-simulation/post-simulation to measure change in their readiness to adopt the new technology.

Results: The scenario was designed and pilot tested before implementation at two rural EDs. There were 60 interprofessional participants across the 2 sites, with 58 pre-simulation and 59 post-simulation surveys completed. The post-simulation mean ratings for each readiness measure (feasibility, quality, resource availability, role clarity, staff receptiveness, and tech usability) increased significantly as a result of the simulation experience.
\end{abstract}

Conclusions: A novel 3-stage simulation-debriefing structure positively targets factors influencing the adoption of new healthcare technologies.

Keywords: Healthcare simulation, Telehealth, Debriefing, Sepsis, Interprofessional simulations, Health information technology, Implementation research

\footnotetext{
*Correspondence: kimberly.l.cooley@osfhealthcare.org

This was a collaborative effort between verbiage to Jump Simulation (an OSF

Healthcare and University of Illinois College of Medicine at Peoria

Collaboration), OSF ConstantCare elCU, and Northwestern University

Feinberg School of Medicine.

'Jump Simulation (an OSF Healthcare and University of Illinois College of

Medicine at Peoria Collaboration), OSF HealthCare and University of Illinois

College of Medicine, 1306 N. Berkeley Avenue, Peoria, IL, USA

Full list of author information is available at the end of the article
}

(c) The Author(s). 2020 Open Access This article is licensed under a Creative Commons Attribution 4.0 International License, which permits use, sharing, adaptation, distribution and reproduction in any medium or format, as long as you give appropriate credit to the original author(s) and the source, provide a link to the Creative Commons licence, and indicate if changes were made. The images or other third party material in this article are included in the article's Creative Commons. licence, unless indicated otherwise in a credit line to the material. If material is not included in the article's Creative Commons licence and your intended use is not permitted by statutory regulation or exceeds the permitted use, you will need to obtain permission directly from the copyright holder. To view a copy of this licence, visit http://creativecommons.org/licenses/by/4.0/ The Creative Commons Public Domain Dedication waiver (http://creativecommons.org/publicdomain/zero/1.0/) applies to the data made available in this article, unless otherwise stated in a credit line to the data. 


\section{Introduction}

Healthcare simulation has served a variety of functions in support of patient safety including latent threat identification [1-3] training for high-criticality/low-frequency events [4], and improving teamwork, [5-7] invasive procedural safety [8], and critical care skills [9]. Less is known about the influence of simulation on the implementation of new technologies in the healthcare setting. While there has been a call to more consistently utilize clinical simulation for testing usability of health information technologies (HIT) [10], subsequent research has focused on the use of simulation for training in preparation for a software HIT implementation [11].

Predictors of technology adoption across industries have most often been studied using variations of the Technology Acceptance Model (TAM) [12], which defines acceptance as intent to use primarily based on perceived usefulness and ease-of-use [13]. However, the TAM's fit with respect to HIT adoption has been questioned [14]. Recent studies in implementation science around clinician healthcare behaviors suggest that knowledge, while a pre-requisite for action is less correlated with simulation-based behavior change than individual beliefs around risk perception, self-efficacy, and intent to implement [15-17]. Given the complexity of change implementation in the healthcare environment, a Consolidated Framework for Implementation Research (CFIR) was proposed in 2009 [18]. This framework synthesized available implementation models with a primary focus on health services. The CFIR divides the factors that influence uptake of a new health services intervention into five domains: intervention characteristics, outer setting, inner setting, characteristics of individuals, and implementation process. Each of these domains encompasses multiple constructs. Figure 1 lists the constructs of the first four domains; all of which provide the context for the implementation process.

In situ simulation affords a unique opportunity to address these domains due to its ability to explore individual use of a HIT intervention within the settings and processes of the system. Debriefing, considered to be the phase of simulation where primary learning and reflection occurs $[19,20]$, also represents an opportunity to explore perceived barriers to HIT use and address them in a social context. The closest analogs in the literature are those in situ simulation efforts that describe the testing of new spaces to detect operational and safety issues [2, 21-23].

In this report, we describe a "3-Act-3-Debrief" in situ simulation structure designed to specifically support implementation of a new HIT process and technology-the use of a telemedicine cart in the care of patients with severe sepsis or septic shock in a rural emergency department (ED). While the success of "in-simulation" debriefing triggered by learner errors has been reported previously $[24,25]$, division of a single-simulated patient encounter into distinct phases that target HIT implementation factors is a novel approach.

The simulated encounter we describe was created as a component of a larger study evaluating the impact of telehealth use in the care of septic patients in two rural emergency departments within a 13-hospital healthcare system. The primary focus of this report is to describe a novel simulation structure that supports implementation of new healthcare technologies. As supporting evidence, the impact of the simulation utilizing the 3-Act-3-Debrief structure was measured by participants' change in individual-related HIT implementation factors from the CFIR: perceived ease of use, feasibility, role clarity, and staff receptiveness.

\section{Methods}

\section{HIT context}

The specific HIT being introduced was a mobile telehealth cart that provided video teleconferencing capability between the ED and the eICU within the hospital system's tertiary care center. The eICU is staffed 24 hours a day by critical care nurses; an intensivist physician is also present overnight to provide support for the eICUs across the healthcare system. Prior to cart introduction, the eICU critical care nursing staff interacted with ED staff solely through telephone contact, and there was no direct eICU interaction with ED patients.

The telehealth carts to be deployed in these EDs were to connect local ED nurses with the electronic intensive care unit (eICU) at the system's tertiary care site as well as provide the option of video-based monitoring of patients with severe sepsis or septic shock.

\section{Scenario design}

The scenario was designed to introduce the HIT in a manner that targeted simulation-accessible constructs within the domains of the CFIR: (1) patient needs, (2) structural characteristics, (3) networks and communications, (4) implementation climate, (5) complexity, (6) relative advantage, (7) knowledge and beliefs, and (8) self-efficacy in use of the intervention as perceived by staff members [18].

As shown in Fig. 2, not all constructs within the framework are amenable to simulation-based support. Within the outer setting domain, it is the patient needs construct that defines the clinical content. In our context, the treatment of sepsis was a strategic target based on sepsis bundle [26] compliance and mortality rates below desired targets. The inner setting constructs reflect the relevant characteristics of the organization where the HIT is being implemented. Simulation cannot directly impact the existing structural characteristics, 


\begin{tabular}{|c|c|c|}
\hline Outer Setting & Patient Needs & \\
\hline \multirow{3}{*}{$\begin{array}{l}\text { Factors external to } \\
\text { the organization }\end{array}$} & Cosmopolitanism & \\
\hline & Peer (Market) Pressure & \\
\hline & External Incentives & \\
\hline Inner Setting & Structure & \\
\hline \multirow{4}{*}{$\begin{array}{l}\text { Characteristics of the organization } \\
\text { implementing the intervention }\end{array}$} & Networks / Communication & \\
\hline & Culture & \\
\hline & Climate & \\
\hline & Readiness & \\
\hline Individual & Knowledge \& Beliefs & \\
\hline \multirow{4}{*}{$\begin{array}{l}\text { Characteristics of the } \\
\text { individual/team/unit/service line } \\
\text { that influence implementation } \\
\text { of intervention }\end{array}$} & Self-Efficacy & \\
\hline & Stage of Change & \\
\hline & Identification with Organization & \\
\hline & Personal Attributes & \\
\hline Intervention & Intervention Source & Outer Setting \\
\hline \multirow{7}{*}{$\begin{array}{l}\text { How the intervention intersects } \\
\text { with individual perception and } \\
\text { context of the inner setting }\end{array}$} & Evidence Strength \& Quality & Inner Setting \\
\hline & Relative Advantage & \\
\hline & Adaptability & Individual \\
\hline & Trialability & \\
\hline & Complexity & 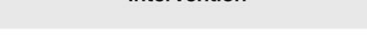 \\
\hline & Design Quality \& Packaging & Compressed version of CFIR \\
\hline & Cost & framework, as seen in Figure 2 \\
\hline
\end{tabular}

which includes 'internal division of labor', nor networks and communications, which refers to existing modes of intraorganizational connection and communication. However, by designing the simulation to be interprofessional and in situ, the concepts of team roles and communication processes could be intentionally explored in the debriefings as the HIT was introduced in its realworld work team context.

Simulation experiences can more directly target the domains of intervention characteristics (complexity, relative advantage), and characteristics of individuals (knowledge and beliefs, self-efficacy) as well as the third inner setting construct of implementation climate because they all are influenced by the interactions of the individual, team, and/or unit with the HIT in the work context. For this project, the simulation was designed to provide the opportunity to train on functionality while directly using the telehealth cart in all relevant portions of workflow. The in situ simulation provides the opportunity for staff to directly experience the relative ease of deployment and where the technology could provide added benefit for patient care.

Prior to implementation, the simulation session was tested at the healthcare system's simulation center with participation from Simulation Operations Specialists, SPs, ED physicians, nurses from the rural sites, telehealth on-site support team, and eICU nurses participating via the cart as they would during in situ simulations. This helped ensure that insights into the new process from all relevant clinical team members could be included in the scenario construction.

Initial pilot testing with ED clinical staff used a traditional scenario design - a simulated patient presenting in septic shock. This approach did not trigger 

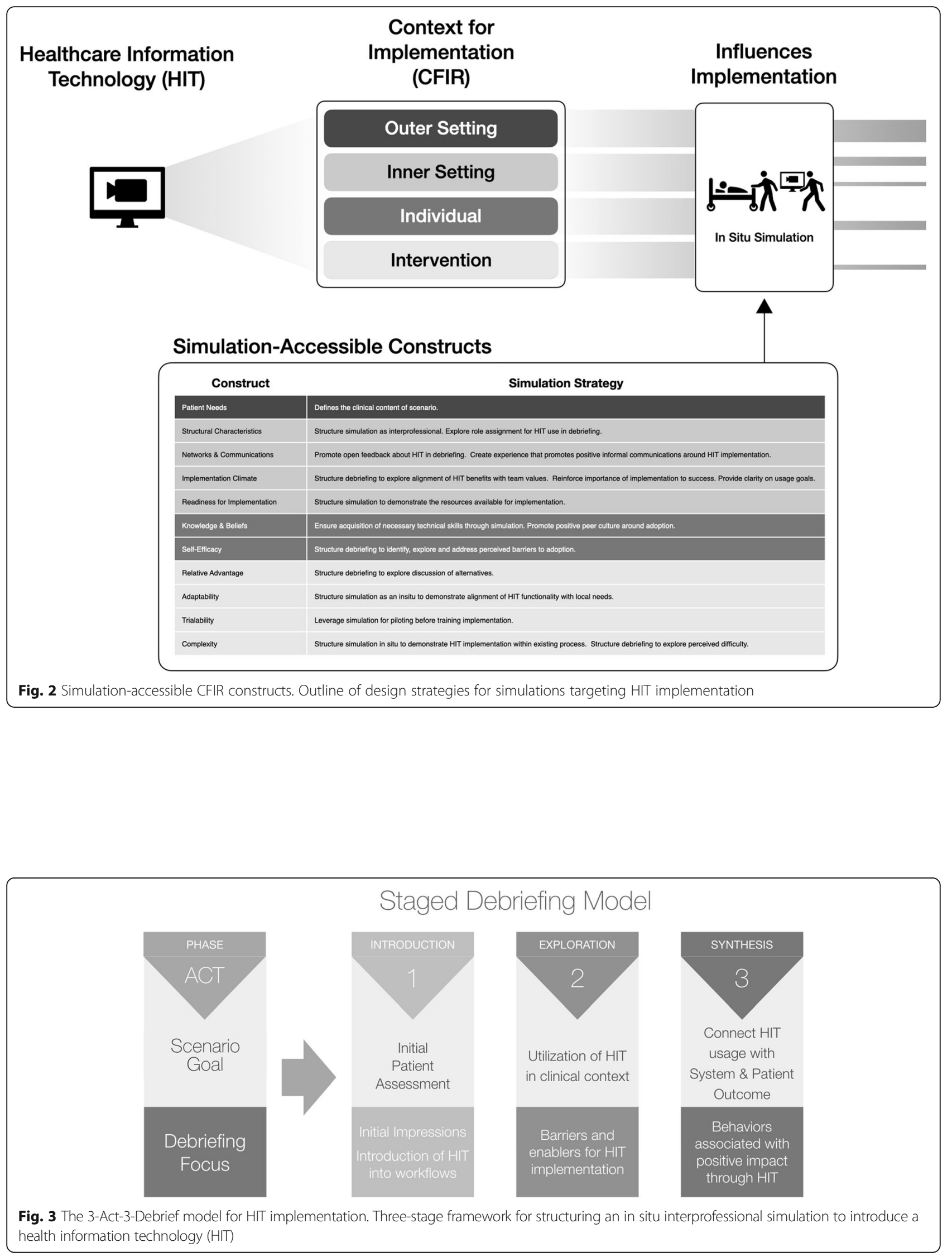
consideration of the telehealth cart despite targeted briefing. Though the constructs were explored in a conversational structure for debriefing [27] the single-stage simulation encounter failed to provide opportunity to experience the HIT's intersection with constructs of intervention complexity, relative advantage, knowledge and beliefs nor self-efficacy.

To facilitate the opportunity for more deliberate reflection and exploration of the targeted CFIR factors in the context of ED workflow, the presentation and clinical progression of the patient was subsequently divided into three "acts", each with its own debrief (Fig. 3). The objectives in the scenario included both HIT implementation and clinical targets. Clinical content for the sepsis patient presentation and progression was based on Centers for Medicare and Medicaid Service's (CMS) definitions and bundles of care for severe sepsis and septic shock [26] so that the team would need to progress through the full course of care during the encounter. A standardized patient (SP) was roomed as a new ED patient, initially hemodynamically stable, with progressive deterioration to begin each subsequent act. Telehealth staff, who were remote at the other end of the telehealth connection, engaged as scripted participants.

The final content summary of each act, as structured for both ED and telehealth teams, is outlined in Table 1. Corresponding debriefing around the HIT as mapped to the targeted implementation constructs is summarized in Table 2.

\section{Additional materials}

Prior to the simulations, all staff were invited via email to participate in eLearning modules that reviewed the sepsis bundle components and recent system changes to the healthcare system's automated electronic health record (EHR) sepsis best practice alert (BPA) process. The updated BPA incorporated real-time data inputs from available labs and vitals into detection algorithms for potential sepsis. Communication expectations and limitations of telehealth participation were also included in the pre-learning material.

\section{Simulation deployment}

The 3-stage sepsis scenario was performed in situ in the two targeted rural EDs over a 1 month period. Simulations were targeted to the interprofessional teams staffing the system EDs (physician, nurse, and patient care technician). Sessions were led by emergency medicine physicians who were also experienced simulation facilitators with formal training in debriefing.

Participants were either paid for extra time to come in at the beginning or stay at the end of shift (most cases) or were relieved during their shift by paid additional staff. Simulations were performed early in the morning to catch night shift staffers at the end of their shifts, then for day shift, and staying into the late morning and early afternoon for middle shift employees. Interprofessional teams typically included one physician provider and 3-5 ED nurses and/or technicians. Nursing participation was

Table 1 Simulation scenario summary. Expected activities to be completed by the ED teams and embedded elCU staff during the in situ simulation

\begin{tabular}{|c|c|c|c|}
\hline Stage & $\begin{array}{l}\text { Time } \\
\text { (min) }\end{array}$ & ED team & Telehealth \\
\hline $\begin{array}{l}\text { Act } \\
\text { One }\end{array}$ & $5-10$ & $\begin{array}{l}\text { 1. At bedside: full team } \\
\text { 2. MD-perform H\&P } \\
\text { 3. RN/tech-pt on monitor, draw labs, IV start }\end{array}$ & $\begin{array}{l}\text { 1. Monitor cart Off } \\
\text { 2. Brief introduction to staff when cart turned on for practice } \\
\text { (in debrief) } \\
\text { 3. Identify ED room number } \\
\text { 4. No clinical discussions }\end{array}$ \\
\hline
\end{tabular}

Transition: Pause for debriefing after sepsis workup initiated

Act $\quad 5-10 \quad$ 1. Telehealth calls bedside RN alerting him/her to BPA firing

Two 2. Team pulls cart to just outside of room, turns it on

3. Clinical introductions

4. Reviews bundle with telehealth, additional orders/interventions

Transition: Pause for debriefing after bundle elements addressed by telehealth team

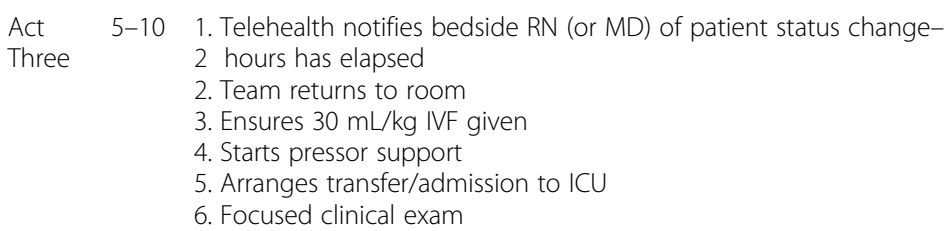

1. Telehealth nurse calls bedside nurse to alert him/her that sepsis BPA has fired on patient 2. Clinical introductions

3. Ask about bundle elements (elCU kept aware via Skype technology ${ }^{24}$

4. "What sources of infection have you considered?" 5. Recommend $30 \mathrm{cc} / \mathrm{kg}$ amount which is approximately $3 \mathrm{~L}$ for the $100 \mathrm{~kg} \mathrm{SP}$

1. Verify bundle elements as needed 2. Verify classification of patient as septic shock 3. If patient admitted kept in regional ICU, emphasize eICU presence

4. Repeat lactate? 
Table 2 HIT debriefing for implementation using the Consolidated Framework for Implementation Research (CFIR) Framework

\begin{tabular}{|c|c|c|c|}
\hline & Goal & Debriefing outline & CFIR domain [construct] \\
\hline \multirow{7}{*}{$\begin{array}{l}\text { Act } \\
\text { One }\end{array}$} & \multirow{7}{*}{$\begin{array}{l}\text { Introduce HIT in the } \\
\text { context of usual } \\
\text { workflow }\end{array}$} & Ensure shared mental model of septic patient & Outer setting [Patient needs] \\
\hline & & $\begin{array}{l}\text { For patients at risk for deterioration, telehealth can help } \\
\text { team observe the patient using the cart. What is the } \\
\text { reality of the workflow at this point? }\end{array}$ & $\begin{array}{l}\text { Inner setting [Structural Characteristics, } \\
\text { Implementation Climate] }\end{array}$ \\
\hline & & Explore roles-who would set up the cart? & Intervention [Complexity] \\
\hline & & $\begin{array}{l}\text { Have participants demonstrate physical maneuvers of } \\
\text { cart }\end{array}$ & $\begin{array}{l}\text { Intervention [Complexity] } \\
\text { Individuals [Knowledge and Beliefs] }\end{array}$ \\
\hline & & $\begin{array}{l}\text { Telehealth RN (in person) demonstrates activating } \\
\text { cart-introduces responding elCU nurse }\end{array}$ & $\begin{array}{l}\text { Intervention [Complexity] } \\
\text { Inner setting [Networks and Communications] }\end{array}$ \\
\hline & & Learners demonstrate activating cart & $\begin{array}{l}\text { Intervention [Complexity] } \\
\text { Individuals [Self-Efficacy] }\end{array}$ \\
\hline & & Learners interact with telehealth nurse via cart & Intervention [Complexity] \\
\hline \multirow{5}{*}{$\begin{array}{l}\text { Act } \\
\text { Two }\end{array}$} & \multirow{5}{*}{$\begin{array}{l}\text { Identify anticipated } \\
\text { barriers to } \\
\text { HIT use }\end{array}$} & Ensure shared mental model of severe sepsis & Outer Setting [Patient Needs] \\
\hline & & Explore ED context for using telehealth cart & $\begin{array}{l}\text { Inner Setting [Implementation Climate] } \\
\text { Individuals [Knowledge and Beliefs] }\end{array}$ \\
\hline & & Explore any prior experience with telemedicine HIT & $\begin{array}{l}\text { Intervention [Complexity] } \\
\text { Individuals [Knowledge and Beliefs] }\end{array}$ \\
\hline & & When would this be helpful? & $\begin{array}{l}\text { Inner Setting [Implementation Climate] } \\
\text { Individuals [Knowledge and Beliefs] } \\
\text { Intervention [Relative Advantage] }\end{array}$ \\
\hline & & What would make it difficult? & Intervention [Complexity] \\
\hline \multirow{7}{*}{$\begin{array}{l}\text { Act } \\
\text { Three }\end{array}$} & \multirow{7}{*}{$\begin{array}{l}\text { Explore relevance, } \\
\text { communication } \\
\text { strategies and clinical } \\
\text { pearls }\end{array}$} & Ensure shared mental model of septic shock & Outer Setting [Patient Needs] \\
\hline & & Telehealth interactions: telephone vs video monitoring & Intervention [Complexity] \\
\hline & & Point-of-contact? (MD vs RN) & $\begin{array}{l}\text { Inner Setting [Networks and Communications] } \\
\text { Intervention [Relative Advantage] }\end{array}$ \\
\hline & & $\begin{array}{l}\text { Communication strategies-in front of patient and/or } \\
\text { families? }\end{array}$ & $\begin{array}{l}\text { Inner Setting [Culture] } \\
\text { Individual [Knowledge and Beliefs] }\end{array}$ \\
\hline & & Conflicting views_-how to address (TeamSTEPPS tools) & $\begin{array}{l}\text { Inner Setting [Culture] } \\
\text { Individual [Knowledge and Beliefs] }\end{array}$ \\
\hline & & $\begin{array}{l}\text { The Sepsis Hospital Concept (elCU capabilities and } \\
\text { limits) }\end{array}$ & Intervention [Relative Advantage] \\
\hline & & Wrap up: balancing barriers vs benefits & $\begin{array}{l}\text { Intervention [Relative Advantage, Complexity] } \\
\text { Process [Reflect and Evaluate] }\end{array}$ \\
\hline
\end{tabular}

Lists goals, outlines descriptions, and corresponding CFIR domain for each debriefing ACT within the sepsis telehealth in situ simulation

emphasized as primary communication between the ED and the eICU occurs at the nursing level within the system.

On-duty eICU nurses participated in the actual scenario via the telehealth care mechanism, and telehealth support personnel were on site for the in situ simulations, primarily participating in the demonstration of HIT functionality in Act 1. A detailed description of the technical aspects of executing these simulations across remote sites has been described [28].

\section{Data collection and analysis}

Pre-simulation and post-simulation surveys were collected electronically during the in situ event using tablet computers and survey software (Qualtrics $\odot$ 2015, Provo, UT). Five-point (Strongly Disagree to Strongly Agree)
Likert scale questions, whose content aligned with the CFIR constructs targeted in the simulation, were selected from a previously validated telemedicine readiness assessment [29] and modified to reflect the HIT under evaluation (Additional files 1 and 2). All statistical analyses were performed using the open source statistical program $\mathrm{R}$ (version 3.6.1) using a 2-sided alternative hypothesis with a $95 \%$ confidence level. Univariate comparisons between participants' Likert scale ratings on the pre- and post-surveys were analyzed using paired samples $t$ tests at an item level both stratified by ED and combined. Additionally, a linear mixed effects model was used to compare rating between the pre- and posttime frames while controlling for the effect of the ED site and the interaction between time frames. Participant and survey item were also included as random effects to 
account for variance due to repeated measurements of the same subject and item.

\section{Results}

\section{Participation}

A total of 60 clinicians participated in the in situ simulations across both sites. One hundred percent of participants from site 1 and $73 \%$ from site 2 availed themselves of the pre-learning opportunity. All participants consented to participate in the surveys, which were not required to participate in the education. Enrollment targets of $80 \%$ were met for participation at both sites based on nursing participation. On average, the 3-Act-3 Debrief sepsis scenario was completed in $46 \mathrm{~min}$.

\section{Survey results}

There were 59 survey respondents from the two sites. Ratings for the Likert-type items, both individually and mean, increased significantly from pre to post when the data was stratified by site and when it was combined (Fig. 4). Paired sample $t$ tests found ratings were significantly higher on the post survey for all items and when ratings were averaged across items within a participant. The mean overall rating increased significantly from a pre-survey score of $3.82 \pm 0.56$ to a post-survey score of $4.5 \pm 0.48, t(56)=10.52, p<0.001$. The electronic survey did not force validate responses resulting in one participant from site 1 not rating HIT quality of care and one participant from site 2 not rating HIT feasibility. When a linear mixed model was used to control for site and account for the variance due to participant and survey item, the results indicated that both sites increased significantly, although the ratings increased less from pre to post at site 2 (Fig. 5).

\section{Discussion}

Elements of the CFIR were successfully integrated into this in situ simulation-based introduction of a new healthcare technology by using the 3-Act-3-Debrief model to deliberately address specific CFIR constructs in a clinically relevant context. This approach differs significantly from what has traditionally been observed for the introduction of new equipment. These sessions generally take place pre-shift, adjacent to direct patient care

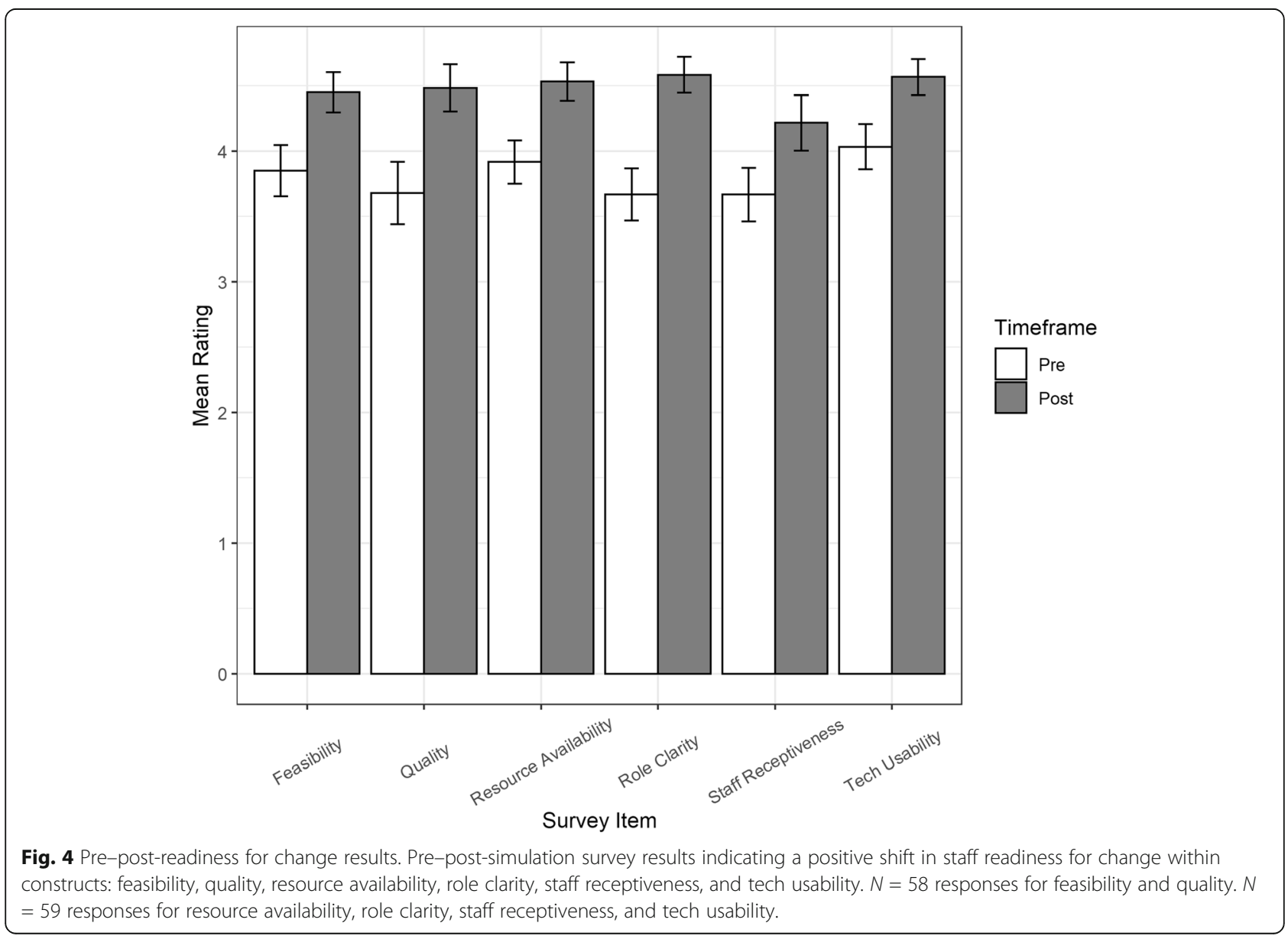




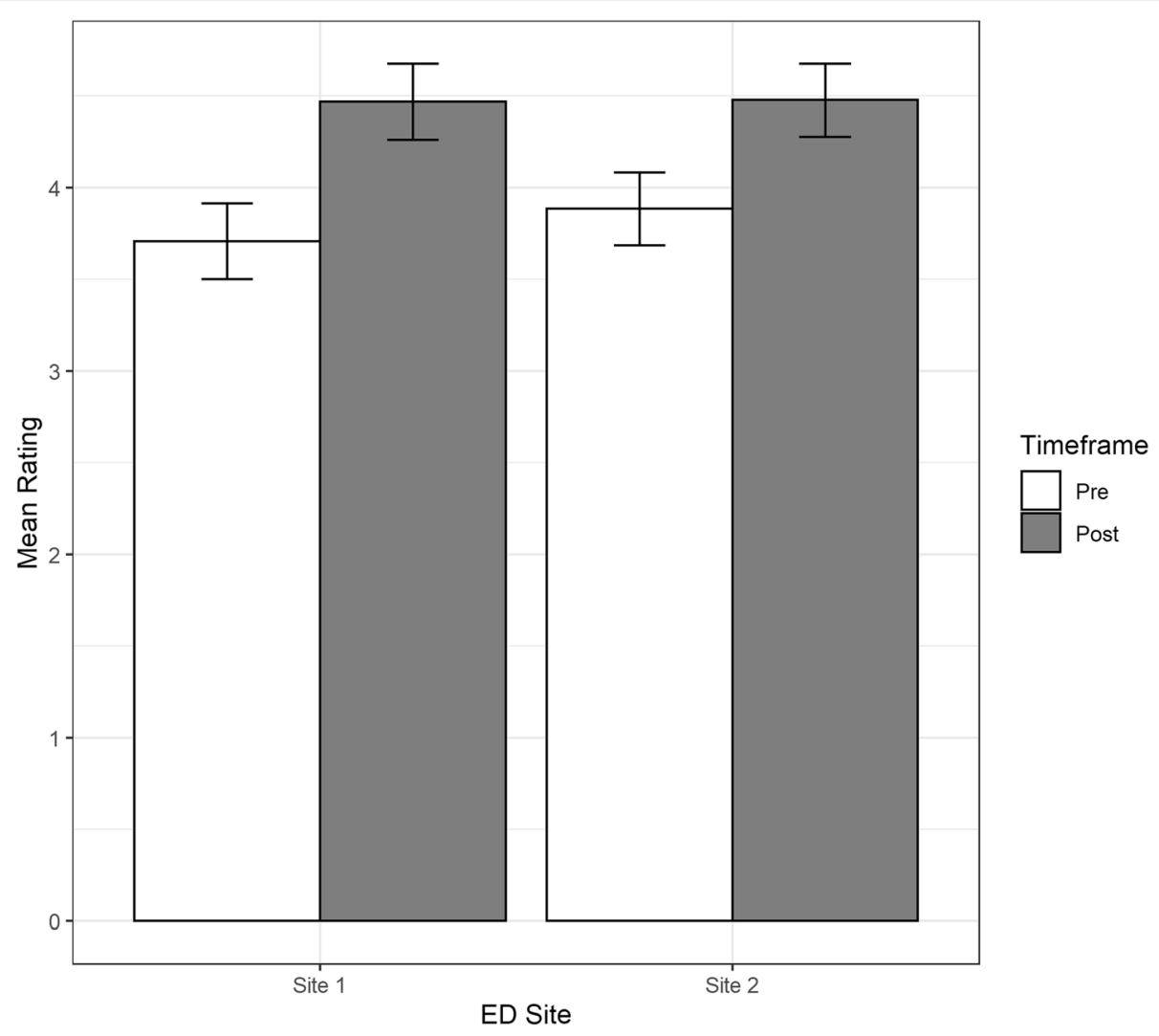

Fig. 5 Pre- and post-survey ratings by site. A linear mixed effects model found the post-simulation timeframe was associated with significantly higher ratings $(b=0.76, \mathrm{SE}=0.11, p<0.001)$ than the pre-simulation time frame at either site. There was also a significant negative interaction between site and timeframe $(b=-0.17, \mathrm{SE}=0.08,0.04)$, which indicates that the ratings increased less from pre to post at site 2 compared to site 1. Although both sites had similar average post-ratings of approximately 4.5 , site 2 had a higher mean pre-rating of $3.88 \pm 0.67$ which increase by 0.6 points from pre to post while site 1 had a lower mean pre-score of $3.71 \pm 0.48$, which increased by 0.76 points from pre to post

areas, and provide supporting background knowledge with brief hands-on practice using the tool. In contrast, the approach described in this report embeds introduction of a new technology within natural workflow through an in situ simulation, thereby respecting the prior experiences of the clinical staff as adult learners who prioritize relevance to their current context and value opportunities to reflect [30].

By structuring the simulation to span the full workflow required to engage the new technology, the structural contexts surrounding its use can be more fully explored. For physician-dependent HIT, such as the electronic health record (EHR), accounting for extrinsic barriers to implementation has been recommended [31] including confidence in related organizational infrastructure, technological assistance and training, which have been correlated with clinician intent to use telemedicinerelated HIT [32].

In addition, engaging interprofessional teams in our sepsis-HIT simulation provided opportunity for all debriefings to address implementation concerns in a social context framed as learning conversations. Negative comments were allowed, and, in fact, encouraged by specifically asking for barriers. In this way, key team members had the opportunity to acknowledge concerns, and issues could be addressed prior to implementation.

The survey results immediately post-simulation provided a small but favorable glimpse into the CFIR constructs in the context of caring for a simulated sepsis patient with telehealth as a proposed adjunct. It is possible that site 2 ratings increased less from pre to post than site 1 because site 2 started with higher pre-ratings, and both groups ran into somewhat of a ceiling effect on the post-survey, since ratings were made on a 5-point scale and both sites had mean post-scores of approximately 4.5. As previously reported [28], the retrospective pre-post-mean \pm SD self-efficacy (11-point scale, anchored $0-100 \%)$ in the use of the telehealth cart also increased significantly, from $5.3 \pm 2.9$ to $8.9 \pm 1.1(\Delta 3.5, p$ $<0.05)$. The significant increases in all of these CFIR construct measures following the simulation-based intervention suggest that simulation-based introductions to HIT can positively impact individual perceptions around HIT implementation. 
The intentional division of a simulated patient's clinical course into three stages of simulation and debrief, regardless of learner actions, has also been recently described in the literature for a simulation targeting skill acquisition by medical students [33]. Similar to our study, authors utilized a 3-stage approach with a learning conversation style of debriefing. For those novice learners, the perceived learning experience was equivalent to standard post-simulation debriefing. While these two studies are promising, additional studies are necessary to further characterize the optimal use of this approach.

\section{Limitations}

This simulation-based intervention was not intended to address all implementation considerations outlined within the CFIR. It would be impossible to cover all aspects of an implementation within a simulation of this nature, so not all the factors influencing implementation were addressed. However, this approach addressed many more facets than the traditional in-service that simply demonstrates the technology. Comparison to the usual in-service approach is limited due to lack of systematic data collection after those events.

The guided questions of the debriefing, while somewhat directive, helped bring out concerns of staff that were anticipated by the implementation group. While a fully open-ended approach to debriefing may have reduced biases in perception, time constraints forced the choice of the more guided approach while still addressing barriers and enablers. The use of physicianinvestigator facilitators may have limited candid sharing of negative perceptions if there was a perceived power gradient despite course debriefers leaving the room for the survey.

The ultimate success of any HIT implementation relies upon elements of the CFIR being addressed during the implementation and early adoption phase. The scope of the analysis presented here is limited in that it precedes actual implementation. The positive shifts in perceptions are necessary and valuable, but the degree to which actual adoption occurs involves more factors than could be addressed through these simulation events and is the focus of future study. Qualitative analysis of the debriefings, post-implementation focus groups, and interviews is ongoing and will be presented in future publications as will the impact of this HIT intervention on patient care outcomes.

\section{Conclusion}

Adoption of new health information technology (HIT) may be positively impacted through leveraging in situ simulation to introduce the new platforms. The 3-Act-3Debrief structure allows for incorporation of key constructs from the Consolidated Framework for Implementation Research (CFIR) that are correlated with successful healthcare implementations. Participation in such a structured in situ simulation around the use of a telehealth cart to improve sepsis care in rural EDs positively impacted selected CFIR measures predictive of subsequent technology adoption.

\section{Supplementary information}

Supplementary information accompanies this paper at https://doi.org/10. 1186/s41077-020-00145-x.

Additional file 1. Pre-survey. Survey questions administered to the rural ED team before the insitu sepsis simulation.

Additional file 2. Post-survey. Survey questions administered to the rural ED team after the in situ sepsis simulation.

\section{Abbreviations}

BPA: Best practice alert; CFIR: Consolidated Framework in

Implementation Research; CMS: Centers for Medicare and Medicaid Services; ED: Emergency department; elCU: Electronic intensive care unit; EHR: Electronic health record; HIT: Health information technology; SP: Standardized patient

\section{Acknowledgements}

The authors would like to acknowledge simulation specialist, Dustin Holzwarth; Jump Simulation Research Assistant Chase Salazar; the Standardized Participant Program at Jump Simulation; OSF ConstantCare elCU staff: William Tillis, MD, Toni Bortell, RN; Sarah Metzger, RN; Lori Nelson, RN; Courtney Pilat, RN; Erika Shaw, RN, and Vicki Weatherington, RN; Rural ED leadership: Erin Nimbler, RN and Lisa Sopher, RN; and all other OSF Saint James ED and OSF St. Mary's ED staff who participated and provided feedback during the study. For the manuscript, we also acknowledge Kyle Formella, MS for the graphics designed to visually convey the concepts presented.

\section{Authors' contributions}

$L B, W B$, and EP designed the study protocol. $L B, W B, A V$, and JV developed, pilot tested, and revised the simulation scenarios. $L B, W B, A V$, and EP facilitated the debriefing. KC coordinated study efforts and participated as observer, providing situational awareness to the elCU during the simulation. AV and KC recruited study participants. JM provided statistical analysis. All authors wrote, read, and approved the final manuscript.

\section{Funding}

This study has been funded by Agency for Healthcare Research and Quality Grant 1 R18 HS024027-01 "In-situ Simulation for Adoption of New Technology to Enhance Safety in Rural EDs".

Availability of data and materials

De-identified survey data generated or analyzed during this study are available from the corresponding author on reasonable request.

Ethics approval and consent to participate

The Peoria Institutional Review Board has reviewed and approved this study, IRB Net \#833011. All research participants were consented prior to study activities. Individual patient data is not applicable for this study.

Consent for publication

Not applicable.

Competing interests

The authors declare they have no competing interests.

Author details

1Jump Simulation (an OSF Healthcare and University of Illinois College of Medicine at Peoria Collaboration), OSF HealthCare and University of Illinois College of Medicine, 1306 N. Berkeley Avenue, Peoria, IL, USA. Department 
of Emergency Medicine, OSF HealthCare, University of Illinois College of Medicine, Peoria, USA. ${ }^{3}$ Department of Emergency Medicine, Northwestern Memorial Hospital, Northwestern University Feinberg School of Medicine, Chicago, USA.

\section{Received: 4 March 2020 Accepted: 1 September 2020}

Published online: 25 September 2020

\section{References}

1. Blike GT, Christoffersen K, Cravero JP, Andeweg SK, Jensen J. A method for measuring system safety and latent errors associated with pediatric procedural sedation. Anesth Analg. 2005;101(1):48-58.

2. Geis GL, Pio B, Pendergrass TL, Moyer MR, Patterson MD. Simulation to Assess the Safety of New Healthcare Teams and New Facilities. Simul Healthc. 2011;6(3):125-33.

3. Kobayashi L, Shapiro MJ, Sucov A, Woolard R, Boss RM III, Dunbar J, et al Portable advanced medical simulation for new emergency department testing and orientation. Acad Emerg Med. 2006;13(6):691-5.

4. Ziv Stephen D. Small Paul Root Wo A. Patient safety and simulation-based medical education. Med Teach. 2000;22(5):489-95.

5. Hunt EA, Hohenhaus SM, Luo X, Frush KS. Simulation of pediatric trauma stabilization in 35 North Carolina Emergency Departments: identification of targets for performance improvement. Pediatr Am Acad Pediatr. 2006;117(3): 641-8.

6. Weaver SJ, Salas E, Lyons R, Lazzara EH, Rosen MA, DiazGranados D, et al. Simulation-based team training at the sharp end: A qualitative study of simulation-based team training design, implementation, and evaluation in healthcare. J Emerg Trauma Shock. 2010;3(4):369-77.

7. Capella J, Smith S, Philp A, Putnam T, Gilbert C, Fry W, et al. Teamwork training improves the clinical care of trauma patients. J Surg Educ. 2010; 67(6):439-43.

8. Wayne DB, Barsuk JH, O'Leary KJ, Fudala MJ, McGaghie WC. Mastery learning of thoracentesis skills by internal medicine residents using simulation technology and deliberate practice. J Hosp Med. 2008;3(1):48-54.

9. Wayne DB, Butter J, Siddall VJ, Fudala MJ, Wade LD, Feinglass J, et al. Mastery learning of advanced cardiac life support skills by internal medicine residents using simulation technology and deliberate practice. J Gen Intern Med. 2006;21(3):251-6.

10. Kushniruk A, Nohr C, Jensen S, Borycki EM. From usability testing to clinical simulations: bringing context into the design and evaluation of usable and safe health information technologies. Contribution of the IMIA Human Factors Engineering for Healthcare Informatics Working Group. Yearb Med Inform. 2013;8:78-85.

11. Richie E, Dove C, Crowe SL, DeArment V, Manwiller J, Wallace M, et al. Utilization of simulation for the introduction of new software technology to the clinical setting. J Patient Saf. 2014;10(3):176-80.

12. Venkatesh V, Davis FD. A theoretical extension of the technology acceptance model: four longitudinal field studies. Manage Sci INFORMS. 2000;46(2):186-204.

13. Venkatesh V, Morris MG, Davis GB, Davis FD. User acceptance of information technology: Toward a unified view. MIS Q. 2003;27(3):425-78.

14. Holden RJ, Karsh B-T. The technology acceptance model: its past and its future in health care. J Biomed Inform Acad Press. 2010;43(1):159-72.

15. Bonetti $D$, Johnston M, Clarkson JE, Grimshaw J, Pitts NB, Eccles M, et al. Applying psychological theories to evidence-based clinical practice: identifying factors predictive of placing preventive fissure sealants. Implement Sci BioMed Central. 2010;5(1):1-14.

16. Eccles MP, Grimshaw JM, Johnston M, Steen N, Pitts NB, Thomas R, Glidewell E, Maclennan G, Bonetti D, Walker A. Applying psychological theories to evidence-based clinical practice: identifying factors predictive of managing upper respiratory tract infections without antibiotics. Implementation Sci. 2007;2:26. https://doi.org/10.1186/1748-5908-2-26 PubMed PMID: 17683558; PMCID: 2042498.

17. Grimshaw JM, Eccles MP, Steen N, Johnston M, Pitts NB, Glidewell L, et al. Applying psychological theories to evidence-based clinical practice: identifying factors predictive of lumbar spine $x$-ray for low back pain in UK primary care practice. Implement Sci. 1st ed. BioMed Central. 2011;6(1):1-13.

18. Damschroder LJ, Aron DC, Keith RE, Kirsh SR, Alexander JA, Lowery JC. Fostering implementation of health services research findings into practice: a consolidated framework for advancing implementation science. Implement Sci. 5 ed. BioMed Central. 2009;4(1):50.
19. Rudolph JW, Simon R, Raemer DB, Eppich WJ. Debriefing as formative assessment: closing performance gaps in medical education. Acad Emerg Med. 2008;15(11):1010-6.

20. Leblanc VR, Manser T, Weinger MB, Musson D, Kutzin J, Howard SK. The study of factors affecting human and systems performance in healthcare using simulation. Simul Healthc. 2011;6(Suppl):S24-9.

21. Bender J, Shields R, Kennally K. Transportable enhanced simulation technologies for pre-implementation limited operations testing: neonatal intensive care unit. Simul Healthc. 2011;6(4):204-12.

22. Kobayashi L, Parchuri R, Gardiner FG, Paolucci GA, Tomaselli NM, Al-Rasheed $\mathrm{RS}$, et al. Use of in-situ simulation and human factors engineering to assess and improve emergency department clinical systems for timely telemetrybased detection of life-threatening arrhythmias. BMJ Qual Saf. 2013;22(1): 72-83 Available from: http://eutils.ncbi.nlm.nih.gov/entrez/eutils/elink. fcgi?dbfrom=pubmed\&id=23060389\&retmode=ref\&cmd=prlinks.

23. Kobayashi L, Dunbar-Viveiros JA, Devine J, Jones MS, Overly FL, Gosbee JW, et al. Pilot-phase findings from high-fidelity In-situ medical simulation investigation of emergency department procedural sedation. Simul Healthc. 2012:7(2):81-94.

24. Van Heukelom JN, Begaz T, Treat R. Comparison of postsimulation debriefing versus in-simulation debriefing in medical simulation. Simul Healthc. 2010;5(2):91-7.

25. Hunt EA, Duval-Arnould JM, Nelson-McMillan KL. Pediatric resident resuscitation skills improve after "Rapid Cycle Deliberate Practice" training. Resuscitation. 2014;85(7):945-51.

26. Rhodes A, Evans LE, Alhazzani W, Levy MM, Antonelli M, Ferrer R, et al. Surviving sepsis campaign: international guidelines for management of sepsis and septic shock: 2016. Intensive Care Med. 14 ed. Springer Berlin Heidelberg. 2017;43(3):304-77.

27. Sawyer T, Eppich Brett-Fleegler M, Grant V, Cheng A. More than one way to debrief: a critical review of healthcare simulation debriefing methods. Simul Healthc. 2016;11(3):209-17

28. Bond WF, et al. A simple low-cost method to integrate telehealth interprofessional team members during in-situ simulation. Simul Healthc. 2019;14(2):129-36

29. Zapka J, Simpson K, Hiott L, Langston L, Fakhry S, Ford D. A mixed methods descriptive investigation of readiness to change in rural hospitals participating in a tele-critical care intervention. BMC Health Serv Res. 2nd ed. BioMed Central Ltd. 2013;13(1):33.

30. Taylor DCM, Hamdy H. Adult learning theories: Implications for learning and teaching in medical education: AMEE Guide No. 83. Med Teach. Informa UK Ltd. London. 2013;35(11):e1561-72.

31. Yarbrough AK, Smith TB. Technology acceptance among physicians: a new take on TAM. Med Care Res Rev. 2007:64(6):650-72.

32. Gagnon MP, Orruño E, Asua J, Abdeljelil AB, Emparanza J. Using a modified technology acceptance model to evaluate healthcare professionals' adoption of a new telemonitoring system. Telemed J E Health. Mary Ann Liebert, Inc. 140 Huguenot Street, 3rd Floor New Rochelle, NY 10801 USA; 2012 Jan;18(1):54-9.

33. Schober P, Kistemaker KRJ, Sijani F, Schwarte LA, van Groeningen D, Krage $R$. Effects of post-scenario debriefing versus stop-and-go debriefing in medical simulation training on skill acquisition and learning experience: a randomized controlled trial. BMC Med Educ. 2019;19(1):334.

\section{Publisher's Note}

Springer Nature remains neutral with regard to jurisdictional claims in published maps and institutional affiliations.

\section{Ready to submit your research? Choose BMC and benefit from:}

- fast, convenient online submission

- thorough peer review by experienced researchers in your field

- rapid publication on acceptance

- support for research data, including large and complex data types

- gold Open Access which fosters wider collaboration and increased citations

- maximum visibility for your research: over $100 \mathrm{M}$ website views per year

At $\mathrm{BMC}$, research is always in progress.

Learn more biomedcentral.com/submission 